\title{
Intraventricular neurocysticercosis: 18 consecutive patients and review of the literature
}

\author{
Albert C. Cuetter, M.D., ANd Russell J. Andrews, M.D. \\ Divisions of Neurology and Neurosurgery, Texas Tech University Health Sciences Center, El Paso, \\ Texas
}

\begin{abstract}
Object. The prognosis for intraventricular neurocysticercosis (IVNCC) is poorer than that for parenchymal NCC, making prompt diagnosis and treatment especially important. Although active, viable intraventricular cysts produce no reaction in the host; they can cause noncommunicating hydrocephalus, the onset of which is frequently abrupt. With the death of the larva comes a local granulomatous ependymitis, generalized ventriculitis, and meningoencephalitis. The authors report on 18 patients with IVNCC (accrued from a larger case series of 62 patients with NCC treated over an 11-year period), detailing clinical presentation, neuroimaging findings, treatment, and outcome.

Methods. All patients presented with hydrocephalus and/or meningitis. The most valuable diagnostic tests were magnetic resonance imaging of the brain and EITB of serum and/or cerebrospinal fluid. Treatment included albendazole and steroid therapy in all cases, and when necessary in cases requiring urgent or emergency ventriculostomy and/or surgical removal of the obstructing cyst (followed by shunt placement if indicated).

Conclusions. An extensive review of the literature on IVNCC has been prepared, with the goal of providing the reader with the information necessary to diagnose and treat this complex and potentially fatal disease in a timely and effective manner.
\end{abstract}

\section{KEY WORDS • arachnoiditis • epilepsy • hydrocephalus • infection • neurocysticercosis, intraventricular $\bullet$ ventriculoperitoneal shunt}

Intraventricular NCC, the presence of tapeworm cysts inside the cerebral ventricular system, occurs in approximately $30 \%$ of patients with NCC. ${ }^{18,19,32}$ Intraventricular cysts are firmly encapsulated. They may circulate freely throughout the CSF pathways or become attached to the ependyma anywhere in the ventricles, but their predilection is for the occipital horn of the lateral and fourth ventricles. ${ }^{10}$ Intraventricular cysts may be single or multiple, and frequently coexist with multiple parenchymal and subarachnoid cysts. ${ }^{18,19,31}$ Seizures subsequently develop in many patients with intraventricular cysts because of the concomitant parenchymal cysts. ${ }^{31}$ Approximately $30 \%$ of all patients with NCC develop hydrocephalus due to CSF obstruction by intraventricular or subarachnoid lesion. ${ }^{39}$ The larvae prefer well-perfused parenchymal sites; the ventricles become populated with cysts when the parenchyma is filled. A cyst in the fourth ventricle, however, tends to be solitary, without accompanying parenchymal cysts. $^{5,16,52}$

\footnotetext{
Abbreviations used in this paper: $\mathrm{CSF}=$ cerebrospinal fluid; $\mathrm{CT}=$ computerized tomography; EITB = enzyme-linked immunoelectrotransfer blot; ICP = intracranial pressure; IVNCC = intraventricular neurocysticercosis; $\mathrm{MR}=$ magnetic resonance; $\mathrm{NCC}=$ neurocysticercosis; TTUHSC $=$ Texas Tech University Health Sciences Center; VP = ventriculoperitoneal.
}

\section{CLINICAL MATERIAL AND METHODS}

We are conducting an ongoing study of NCC at TTUH$\mathrm{SC}$ in El Paso, a metropolitan area of 1 million citizens on the United States-Mexico border, to determine 1) its clinical presentation; 2) its most appropriate neuroimaging and laboratory diagnostic tests; and 3) its optimum management, particularly in cases of IVNCC.

Since June 1990, we have identified 62 cases of NCC at TTUHSC and its affiliate, Thomason Hospital, also in El Paso. Seven patients were identified retrospectively and 55 prospectively. Data are analyzed with the aid of an SPSS 6.1 spreadsheet program (SPSS, Chicago, IL). This series included all patients who presented at TTUHSC and Thomason Hospital and in whom the diagnosis of active and inflammatory NCC was made. Patients with only inactive calcified lesions were not included. Patient information includes age, sex, demographic data, clinical presentation, neuroimaging findings, immunodiagnostic results, treatment, and clinical outcome. Multiple admissions and visits were counted only once.

\section{RESULTS}

Of the 62 patients with NCC, $70 \%$ were male, all were Hispanic, approximately one half were natives of El Paso, and the mean age was 35 years (range 16-74 years). 


\section{Clinical Presentation}

Symptoms on presentation correlated with the location of the cysts. Epileptic seizures (partial simple, complex, and secondarily generalized seizures) occurred in 46 patients (74\%). All patients harboring single inflammatory parenchymal cysts (25 cases) and 21 of 35 patients harboring multiple parenchymal, subarachnoid, and intraventricular cysts presented with epileptic seizures. Of the 35 patients with multiple lesions, 16 (46\%) also harbored intraventricular cysts. In two of the 18 cases with IVNCC, single or multiple fourth ventricular cysts were present without parenchymal cysts, and in 16 cases, intraventricular cysts were present in combination with parenchymal cysts. Hydrocephalus was present in all patients with intraventricular cysts, and all presented with symptoms of increased ICP and/or meningitis. Three patients with intraventricular cysts also suffered epileptic seizures. Two patients with multiple parenchymal cysts who presented with seizures also experienced mild hemiparesis.

\section{Neuroimaging Studies}

All patients underwent CT or MR imaging of the brain, the latter being the imaging modality of choice. Examples are given in the figures, and we further detail imaging characteristics in the Discussion section.

\section{Immunodiagnostic Studies}

Enzyme-linked immunosorbent assay of serum was performed in 33 patients; however, this test was discontinued in 1998 because the rate of positive findings was only $55 \%$ (18 patients). Enzyme-linked immunoelectrotransfer blot of serum and CSF, obtained by lumbar puncture or ventriculostomy, was performed in 35 patients. Results for NCC were positive in $80 \%$ of patients: nine of nine with intraventricular cysts, seven of seven with subarachnoid cysts and meningitis, and 11 of 19 with parenchymal cysts. In nine patients with intraventricular cysts, serum EITB and brain neuroimaging findings were considered sufficient for establishing the diagnosis. ${ }^{1,42,50}$

\section{Tissue Diagnosis}

In two patients, specimens were obtained from a single parenchymal lesion; in another two patients, specimens were obtained from single or multiple fourth ventricular cysts. In four patients, specimens were obtained from multiple cysts in the posterior fossa and in six, specimens were obtained through ventriculostomy. All tissue findings were positive for NCC.

\section{Treatment and Outcome}

Treatment was symptom specific and varied according to several factors: 1) location and stage of evolution of the cysts; 2) clinical status of the patient; 3) patient's preference; 4) presence of hydrocephalus; and 5) attending physician's preference and experience. Treatment included administration of anthelmintic drugs (all patients received albendazole; praziquantel was not used in this series) in combination with steroid agents, emergency ventriculostomy, resection of the cysts, VP shunt placement, and combinations of these methods.
In 10 of the 18 patients a VP shunt was placed. In three of these 10 viable fourth ventricular cysts were resected, and in two others the cysts were excised from elsewhere in the ventricular system. Six of the ten VP shunts required revision within 3 years. Two patients died of progressive hydrocephalus despite having undergone shunt placement.

In the eight patients with minimal or no hydrocephalus in whom initial treatment did not include shunt placement, the cysts resolved with albendazole treatment. In one patient, however, hydrocephalus developed 2 years later due to adhesions and scarring, and subsequent treatment with a VP shunt was successful. One patient with an asymptomatic cyst died of acute hydrocephalus following a vaginal hysterectomy; in Juarez, Mexico another patient died of meningitis after undergoing the placement of a VP shunt to treat hydrocephalus that developed 1 year after initial diagnosis and treatment at TTUHSC.

\section{DISCUSSION}

The development of an active cysticercus, from the time the eggs are ingested, is completed within 60 to 70 days. ${ }^{6}$ Cysts can be parenchymal, subarachnoid, or intraventricular. A viable larva in an active-phase cyst can live up to 7 years. $^{6}$

A useful classification of NCC is that proposed by Carpio, et al.; ${ }^{11}$ active (vesicular, viable), inflammatory (involutional, transitional, colloidal), and inactive lesions. Active cysts usually do not cause clinical symptoms unless located in areas of the brain where they may cause seizures or hydrocephalus. Active intraventricular cysts may persist for years but become symptomatic if they obstruct CSF flow, with consequent hydrocephalus and increased ICP. The lateral ventricles and the fourth ventricle are the most common locations for IVNCC.

Intraventricular cysts have a more aggressive behavior than their parenchymal counterparts. Symptoms associated with the parenchymal lesions largely result from the host's inflammatory response to the dead or dying larva; irritation and edema of the brain and epileptic seizures may develop.

Intraventricular cysts may become symptomatic at the time of implantation secondary to obstruction of CSF flow, with consequent hydrocephalus and the symptoms and signs of increased ICP. When involution begins, the inflammatory reaction around a dead or dying cyst produces ependymitis, scarring, obstruction, and ventriculitis.

\section{Clinical Presentations}

Parenchymal Cysts. Parenchymal symptomatic NCC is largely a result of host inflammatory response, usually presenting, as in our series, with epileptic seizures. A good prognosis is associated with parenchymal inflammatory disease. The secondary symptomatology of coexisting parenchymal involutional cysts may lead to the discovery of asymptomatic intraventricular cysts with or without hydrocephalus. Thus, there are patients who present with tonic-clonic generalized seizures or partial seizures secondary to parenchymal involutional cysts, in whom intraventricular asymptomatic cysts are also present (Fig. 1). 


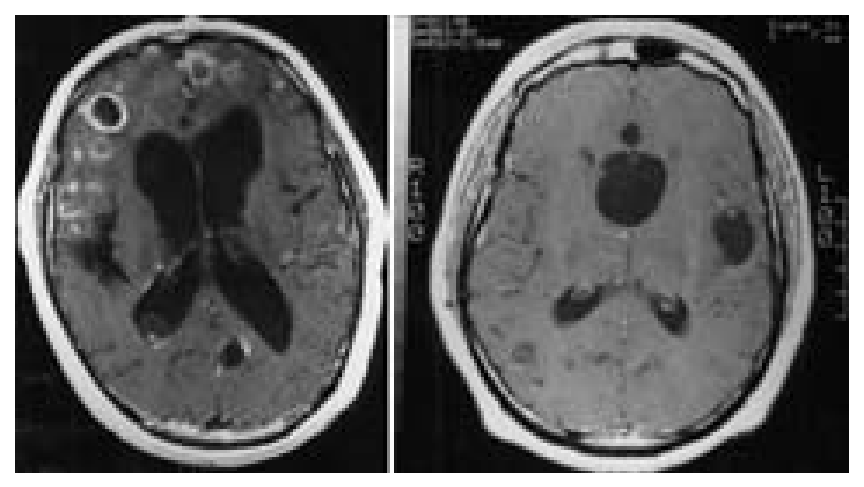

Fig. 1. Left: Axial $\mathrm{T}_{1}$-weighted postcontrast MR image revealing multiple parenchymal cysts and an intraventricular cyst in the occipital horn of the right lateral ventricle. This 32-year-old man presented with seizures. The hydrocephalus had not yet produced symptoms. Right: Axial $\mathrm{T}_{1}$-weighted postcontrast MR image demonstrating a cyst in the septum pellucidum and active parenchymal cysts.

Obstruction of CSF Flow-Related Symptoms. Cysts may cause either a gradual or an abrupt obstruction of CSF flow. Those moving within the ventricles may obstruct one of the vital communication passages, effecting intermittent or permanent blockage of the CSF flow. ${ }^{31}$ Abrupt CSF obstruction results in acute hydrocephalus, in which symptoms and signs include headache, diplopia, dizziness, vomiting, restlessness, seizures, respiratory changes, bradycardia, blood pressure elevation, and alteration of consciousness.

Abrupt intermittent obstruction of CSF flow may last hours to days. Sudden alterations of head position may change the location of the cyst, triggering or alleviate headaches, or producing a fleeting loss of strength or muscle tone. ${ }^{26,31,33}$ These drop attacks may be due to associated sudden bilateral ischemic changes in the brainstem.

Abrupt permanent obstruction may cause acute hydrocephalus leading to stupor, coma, and death due to brain herniation. ${ }^{4,26,31,33,44}$ Sites of obstruction include the foramen of Monro, third ventricle, aqueduct of Sylvius, and fourth ventricle. ${ }^{4}$ Noncommunicating hydrocephalus may require emergency intervention to prevent brain herniation. Direct compression of the brainstem and midline cerebellar structures secondary to fourth ventricular obstruction may produce, in addition to symptoms of increased ICP, focal deficits such as gait ataxia, dysmetria, and diplopia. ${ }^{15,20}$ In children this clinical presentation may resemble that of a midline cerebellar tumor. Obstruction of the aqueduct of Sylvius may produce a superior aqueductal syndrome, in which paralysis of vertical gaze as well as other symptoms and signs of increased ICP occur. ${ }^{47}$

Chronic obstruction can produce an insidious increase in ICP. ${ }^{31}$ If not treated, patients thus afflicted may deteriorate abruptly. ${ }^{33}$ Frequently the obstruction is due to a large cyst in the fourth ventricle. Symptoms include headache, nausea, vomiting, somnolence, memory and behavioral changes, and gait disturbance, and they may be present for several months prior to diagnosis. ${ }^{5,31,33,44}$ Findings include decreased alertness, papilledema, and focal motor deficits, including corticospinal tract signs and frontal lobe motor apraxia. The latter is the result of extensive bilateral le- sions of frontal lobe long motor tracts in the internal capsule that are caused by enlarged lateral ventricles, the ventricular expansion being maximal in the frontal horns.

Obstruction of CSF Flow-and Inflammation-Related Symptoms. Patients who experience symptoms secondary to CSF obstruction and inflammation are the most likely to die of chronic complications. An asymptomatic intraventricular cyst becomes symptomatic when the cyst larva dies, resulting in involution and inflammation. The involutional cyst liberates antigenic substances that cause an inflammatory reaction throughout the ventricular system. This granular ependymitis fixes the cyst capsule to the ventricular wall with strong adhesions and fibrosis. The resulting progressive hydrocephalus and increased ICP may prove fatal (especially in the fourth ventricle), in part because fixed cysts may not be amenable to excision without damage to adjacent brain tissue. ${ }^{32,37,39}$

Patients present with increased ICP, meningoencephalitis (fever, alteration of consciousness, nuchal rigidity), focal neurological deficit, and inflammatory reaction detectable in the CSF ${ }^{29,39}$ Even with provision of appropriate treatment, mental deterioration, blindness, quadriparesis, and ataxia may occur. ${ }^{29}$ Complications include cerebral and brainstem infarction due to angiitis, hypothalamic dysfunction, infections, repeated shunt failure, arachnoiditis, ependymitis, and ventriculitis.

Inflammatory fourth ventricular cysts can be associated with widespread granular ependymitis and ventriculitis. The ventricular enlargement may persist after a VP shunt is placed to treat the hydrocephalus. ${ }^{39}$ There may be single or multiple involutional cysts in the fourth ventricle, but occasionally no cysts are found either on neuroimaging studies or at surgery.

Clinically similar to IVNCC, but more appropriately termed meningeal NCC, is basilar arachnoiditis and leptomeningeal scarring that can cause communicating hydrocephalus. This communicating hydrocephalus can manifest the same symptoms, neurological deficits, and fatal outcome as noncommunicating hydrocephalus.

\section{Neuroimaging and Laboratory Diagnosis}

The diagnosis of IVNCC is based on clinical presentation, MR imaging evidence of cystic lesions containing the scolex, and isolating the parasite histologically from the brain lesions or the $\mathrm{CSF}^{21}$ Because the clinical presentation of IVNCC is nondiagnostic, one must review a patient's history, laboratory findings, and neuroimaging studies to confirm the diagnosis.

Neuroimaging Studies. For several reasons CT scanning may fail to demonstrate small cysts that do not deform the ventricles: 1) they share the same density as CSF; 2 ) the cyst wall and the scolex are not visible; and 3) the cyst does not show contrast enhancement. ${ }^{28,30,52} \mathrm{~A}$ large intraventricular cyst may be seen on CT scans, even without hydrocephalus, if it deforms the ventricle and the cyst is outlined by the normal ventricular cavity. A cyst deforming or enlarging the fourth ventricle is usually well visualized on $\mathrm{CT}$ scans if narrow (3- or 5-mm) slices are obtained. Edema of the adjacent tissues is frequently present if the cyst is in the inflammatory stage (Fig. 2). Computerized tomography scanning may also reveal calcified inactive parenchymal lesions. The CT-documented 


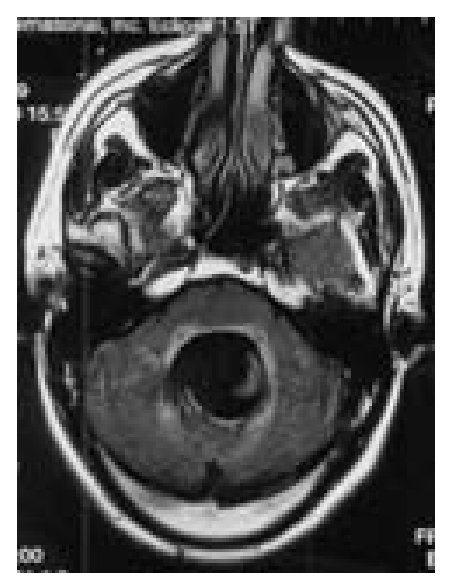

Fig. 2. Axial fluid-attenuated inversion-recovery image revealing an inflammatory cyst in the fourth ventricle, edema in the surrounding brain tissue, and the mural nodule.

diagnosis is facilitated by finding cysts in different stages of evolution, including calcifications, as well as the presence of hydrocephalus, even if the obstructing cysts are not visualized.

Magnetic resonance imaging is the most important neuroimaging study, revealing intraventricular cysts in approximately $80 \%$ of cases. ${ }^{13}$ A viable active intraventricular cyst appears as a spherical lesion of 10 to $20 \mathrm{~mm}$ in diameter on $\mathrm{T}_{1}$-weighted and fluid-attenuated inversionrecovery imaging. The scolex is frequently demonstrated as a mural nodule having the hyperintensity of fat. A cystic lesion with a scolex is one of the absolute diagnostic criteria. ${ }^{24}$ The cyst wall appears as a thin hyperinten-sity between the darkness of the cyst content and the CSF. On $\mathrm{T}_{2}$-weighted imaging, the cyst contents are isointense with the surrounding tissues, and the scolex is hyperintense.

The granular ependymitis that accompanies inflammatory, involutional cysts shows ringlike or nodular enhancement. The inflammatory features of an involutional cyst are demonstrated on $\mathrm{T}_{1}$-weighted MR imaging without gadolinium administration. Features include: 1 ) hyperintense cyst wall (Fig. 3 left); 2) hyperintense scolex (Fig. 3 right); and 3) edema around the cyst (Fig. 3 left). Inflammatory intraventricular cysts have ring enhancement with surrounding edema similar to inflammatory parenchymal cysts visualized on contrast-enhanced $\mathrm{T}_{\text {-weight- }}$ ed MR imaging (Fig. 1 left). ${ }^{52}$ Neuroimaging alone may not be sufficient to differentiate ring-enhancing IVNCC lesion from a neoplastic or other inflammatory process. ${ }^{52}$

Occasionally there may be hydrocephalus and other MR imaging-documented findings in cases of IVNCC, but no intraventricular cysts are observed. Ventricular dilation may be secondary to ependymitis, scarring, or meningeal involvement. Unilateral ventricular enlargement may result from a cyst in the third ventricle or the foramen of Monro. Fourth ventricular cysts often produce hydrocephalus, but, for unexplained reasons, usually are not accompanied by parenchymal cysts. ${ }^{52}$

Laboratory Studies. The primary immunodiagnostic methods for diagnosing NCC are the EITB and the enzyme-linked immunosorbent assay. ${ }^{1,42,50}$ Whereas these

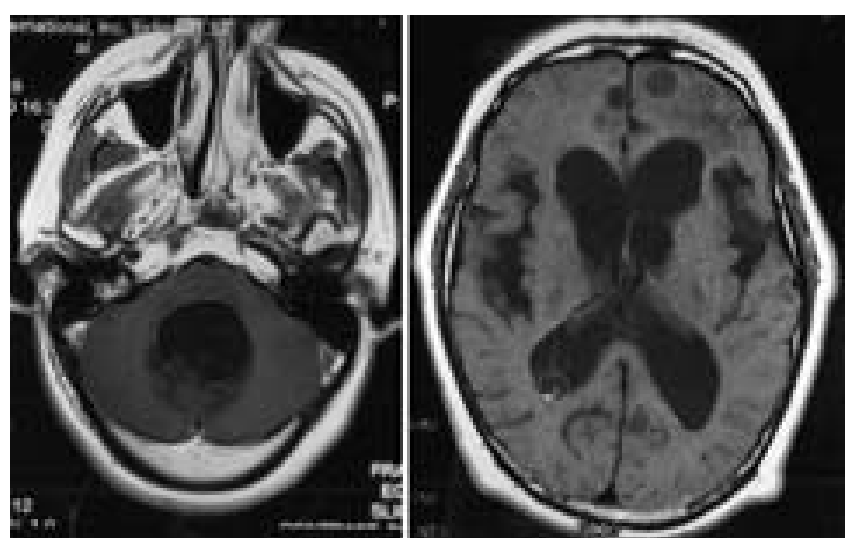

Fig. 3. Axial $\mathrm{T}_{1}$-weighted images. Left: An inflammatory cyst is observed in the fourth ventricle with ring enhancement and edema surrounding the cyst. Right: A cyst is demonstrated in the occipital horn of the right lateral ventricle. The scolex is visible.

tests are highly sensitive in multiple inflammatory lesions, they are not so in those with single viable lesions. In over $80 \%$ of patients with intraventricular inflammatory lesions, EITB assays both are positive for NCC in serum and in CSF, regardless of the number or apparent condition of the cysts. ${ }^{50}$ The enzyme-linked immunosorbent assay has a high rate of both false-positive and false-negative results. ${ }^{1,42}$ Immunodiagnostic tests may yield falsepositive reactions in tuberculosis, as well as in parasitic diseases such as echinococcis. ${ }^{42}$

Lumbar puncture is contraindicated in patients in whom increased ICP is suspected. A ventriculostomy may provide an aid to diagnosis as well as therapeutic drainage. Cerebrospinal fluid abnormalities are directly proportional to the degree of local inflammation and ventriculitis. In $50 \%$ of cases the CSF cell count, protein, and glucose are normal..$^{33}$ In the other $50 \%$ a moderate mixed pleocytosis, increased protein, and hypoglycorrhagia are seen. Pleocytosis is more commonly lymphomonuclear than polymorphonuclear, with eosinophilia occurring in approximately $20 \%$ of cases. ${ }^{9,31,33,44}$ Glucose is reduced in approximately $6 \%$ of cases; protein may be elevated up to $420 \mathrm{mg} / \mathrm{dl} .^{12,44}$

An important diagnostic criterion is histological demonstration of the parasite. The tissue may be obtained from the surgically removed cyst or from intraventricular contents obtained through ventriculostomy. ${ }^{50}$

Differential Diagnosis. The differential diagnosis of IVNCC includes toxoplasmosis, fungal and bacterial meningitis, hydrocephalic sequelae of tuberculous meningitis, echinococcosis, intraventricular neoplasms, and noninfectious granulomatous chronic meningitis. ${ }^{1,16,20,54}$

Toxoplasmosis may present with enlarged ventricles caused by ependymitis and aqueductal stenosis; parenchymal calcifications may resemble cysticercosis ${ }^{30} \mathrm{Although}$ bacterial and coccidioidal ependymitis with ventriculitis can produce hydrocephalus and associated enhancement of the ependymal ventricular walls, the clinical picture combined with the absence of cysts or other signs of NCC make differentiation relatively easy. Granulomatous tuberculous meningitis may present with hydrocephalus, but involvement of the basal meninges on postgadolinium MR imaging is much more common than in IVNCC. ${ }^{8,43}$ 
The multiloculated parenchymal cystic structure of echinococcosis may resemble IVNCC on MR imaging. A third ventricular cysticercal lesion (Fig. 4) may mimic a colloid cyst. ${ }^{51}$ Fourth ventricle cysts may appear as neoplasms such as cystic medulloblastoma, astrocytoma, or ependymoma, with CSF obstruction and edema of adjacent brain (Figs. 2 and 3 left). Characteristics indicating diagnosis of a tumor over IVNCC include the presence of: 1) contrast enhancement; 2) more edema in the adjacent tissues; and 3) extension up and down the fourth ventricle and laterally into the prepontine cisterns. Other conditions such as sarcoidosis and meningeal carcinomatosis, which produce leptomeningitis and pachymeningitis, may also cause chronic hydrocephalus. ${ }^{30}$

\section{Treatment of IVNCC}

The treatment of IVNCC is tailored to the presenting symptoms. The optimum treatment selected from the following therapeutic modalities depends on several factors, including the patient's condition, location of the cysts, and evolutional stage of the cysts: 1) emergency ventriculostomy; 2) placement of a VP shunt; 3) endoscopic or open extirpation of obstructing cysts; 4) antihelmintic medications (albendazole and praziquantel); 5) steroid therapy; and 6) antiepileptic medications.

Acute hydrocephalus usually requires ventriculostomy and subsequent resection of the cysts obstructing CSF

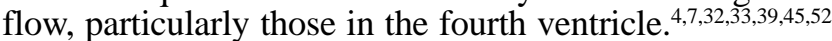
Whether to choose endoscopic or open resection depends on the surgeon's experience; the less invasive endoscopic removal being preferred in most situations.

Involutional inflammatory cysts are attached to the ventricular wall by strong adhesions and cannot easily be removed without damaging adjacent brain tissue., , $, 32,39$ Removal of the cyst does not typically obviate the need for a shunt. ${ }^{4,16}$ Thus, when neuroimaging evidence of ependymitis exists, the preferred treatment is placement of a VP shunt without removal of the cyst.

Although differentiation between a viable and an inflammatory intraventricular cyst is usually difficult, the

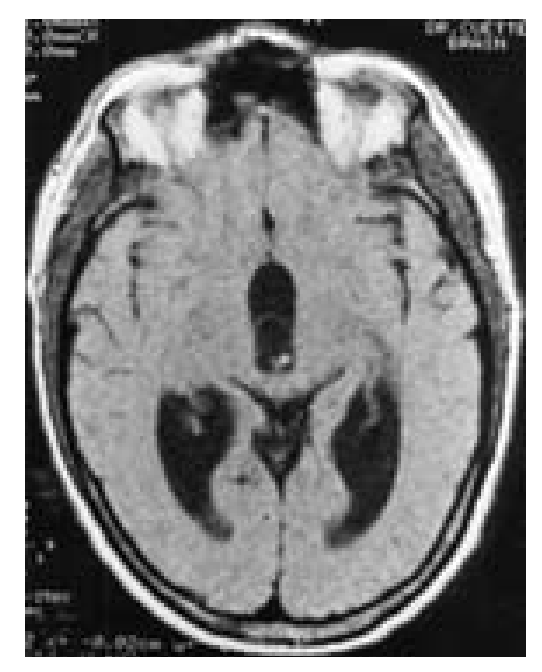

Fig. 4. Axial $\mathrm{T}_{1}$-weighted image revealing a cyst in the third ventricle in a 40 -year-old man presenting with headaches. following may prove helpful. Viable cysts do not provoke symptoms or signs of meningitis, and they produce no or only discrete inflammatory reaction on examination of CSF samples. An involutional inflammatory cyst typically shows symptoms and signs of meningitis, focal neurological deficit, and inflammatory response on examination of $\mathrm{CSF}^{39}$ On $\mathrm{T}_{1}$-weighted noncontrast $\mathrm{MR}$ images, an inflammatory cyst has a hyperintense wall and edema of adjacent brain tissue (Fig. 3 left), whereas on $\mathrm{T}_{1}$-weighted postcontrast MR images there is a ring enhancement in the cyst wall (Fig. 1 upper).

Emergency ventriculostomy, typically followed by insertion of a VP shunt, is indicated in patients with acute hydrocephalus due to intraventricular inflammatory cysts. $^{4,17,53}$ Some benefit may be realized from dexamethasone administered in therapeutic declining-dose therapy. $5,9,31,38$

A VP shunt is usually required in patients with chronic hydrocephalus and increased ICP. ${ }^{14}$ The indications for excision of the viable cyst(s) include the following: 1) significant mass effect; 2) obstruction of CSF flow; 3) shunt placement precluded by the cyst; and 4) uncertain diagnosis.$^{14}$ Fourth ventricular viable cysts should typically undergo extirpation because they may cause brainstem compression even after insertion of a VP shunt. ${ }^{33}$ Standard neurosurgical approaches are performed depending on the cyst location, including 1) transcortical for lateral ventricle cysts; 2) transcallosal or transcortical for third ventricle cysts; and 3) midline suboccipital for fourth ventricle cysts. $4,17,32,51$

The prudent neurosurgeon takes into account the possibility that a cyst may migrate between the time of diagnosis and surgery. ${ }^{23}$ Cyst migration must be ruled out by examining a CT or MR image obtained immediately before surgery. ${ }^{52,53}$ Multiple cysts and multiple obstructions with loculated hydrocephalus may require multiple ventricular catheters to drain the separate loculations. ${ }^{3}$ Patients harboring intraventricular cysts without hydrocephalus require close observation in case acute obstruction occurs and emergency shunt surgery becomes necessary.

Insertion of a shunt remains the primary therapy for inflammatory IVNCC, despite frequent shunt-related complications in these patients. Ventriculoperitoneal shunt obstruction, either by cyst-related gelatinous material or by high CSF protein, is the most common complication. ${ }^{41}$ Early shunt revision should be considered if the patient experiences no improvement after shunt insertion, and recurrent shunt obstruction by cyst material is another reason for excision of intraventricular cysts. $., 14,44$

Although the authors of a recent report have noted success in using anthelmintic drugs without surgery in 33 patients with giant $(>5$-cm-diameter) subarachnoid cysts, the role of anthelmintic therapy in IVNCC remains uncertain. ${ }^{35}$ The use of anthelmintic drugs in conjunction with shunts has been advocated to eradicate viable cysts and to decrease the rate of shunt failures.,44 The use of praziquantel, an isoquinoline with broad anthelmintic activity, is controversial in IVNCC, because earlier results were associated with a poor outcome. ${ }^{49}$ Both therapeutic failures and successes with praziquantel have been demonstrated in the treatment of IVNCC. ${ }^{2,5,19,25,29,34,36,49}$ The recommended dose is $50 \mathrm{mg} / \mathrm{kg} /$ day for 14 days, with concomitant use of dexamethasone. 
Albendazole, a benzimidazole with proven antiparasitic properties, has been used to treat IVNCC successfully. 18,19,22,46 Intraventricular cysts may disappear within 3 months after treatment. ${ }^{18,19,21,46}$ The dose is $15 \mathrm{mg} / \mathrm{kg} /$ day for 15 days. The daily dose is divided into three administrations, with concomitant use of dexamethasone, in two courses given 1 month apart. Intermittent long-term steroid therapy may reduce the incidence of shunt failures. ${ }^{48}$

Intraventricular neurocysticercosis may respond favorably to praziquantel or albendazole, or the favorable response may be due to the natural history of the disease. There is an inflammatory reaction similar to that seen with the natural death of the larva when the cyst larvae die following anthelmintic therapy. ${ }^{12,40}$ The scarring and granulomatous ependymitis may lead to acute hydrocephalus. Some authors therefore have questioned the effectiveness of anthelmintic therapy in IVNCC, even suggesting that such treatment is associated with an increase in long-term sequelae..$^{12}$ To resolve the debate regarding anthelmintic therapy in IVNCC, collaborative clinical trials are needed to evaluate specific medical treatments for IVNCC as well as to develop a better understanding of its clinical course. ${ }^{12,40}$

Two beneficial effects of anthelmintic drugs are the destruction of 1) associated viable parenchymal cysts, and 2) the intestinal parasite that may be a source of recurrent autoinfection.

\section{Prognosis of NCC}

In patients with IVNCC who undergo insertion of a VP shunt or resection of intraventricular cysts improvement typically occurs with resolution of the hydrocephalus. ${ }^{54}$ Intraventricular neurocysticercosis, however, can be fatal with a mortality rate of $13 \%$ in patients with acute hydrocephalus. $^{53}$

Because the mass effect of a cyst in the posterior fossa is less well tolerated than in supratentorial sites, the prognosis for patients with fourth ventricular cysts is guarded. Fourth ventricular cysts usually present in the inflammatory state, with edema and adhesion to the adjacent tissue making resection of the entire lesion difficult. Shunt surgery does not always ensure a good prognosis. ${ }^{44}$

Hydrocephalus recurs in some patients with IVNCC, even after the cysts have disappeared. ${ }^{15,18,19,28}$ Although reinfection occurs occasionally, more commonly the reason is CSF obstruction by chronic adhesions and thickening from involutional cysts. This emphasizes the need for aggressive initial treatment in these patients, including shunt therapy.

\section{CONCLUSIONS}

Intraventricular neurocysticercosis continues to be a potentially fatal condition in which the rate of permanent morbidity is high. Although active, viable intraventricular cysts do not produce a host reaction, they can obstruct CSF flow, leading to the complex clinical syndromes of hydrocephalus. The cysts can migrate throughout the ventricular system, inducing acute intermittent or permanent increased ICP and hydrocephalus. There is a local granulomatous ependymitis and generalized ventriculitis when the larvae die, resulting in hydrocephalus, increased ICP, and meningoencephalitis.
Neuroimaging (CT and MR imaging) remains the most important diagnostic study for IVNCC, especially if cysts in different stages of evolution are found. Enzyme-linked immunoelectrotransfer blot in both serum and CSF is positive in more than $80 \%$ of patients with meningeal irritation due to inflammatory intraventricular cysts.

The treatment of IVNCC is symptom specific. Dexamethasone may alleviate the increased ICP, cerebral edema, and inflammation. Both success and failure have been reported in conjunction with anthelmintic medications (albendazole or praziquantel). These medications hasten the evolution of intraventricular viable cysts, which may trigger an inflammatory response similar to that seen with the natural death of the parasite. Thus, long-term sequelae may result either with or without the use of anthelmintic medications.

Acute hydrocephalus is treated with a ventriculostomy followed by placement of a VP shunt. In patients with chronic hydrocephalus associated with intraventricular cysts, the selection of a VP shunt, resection of the cysts, and anthelmintic therapy may challenge the common sense, experience, and judgment of the most experienced physician.

\section{References}

1. Alarcon F, Duenas G, Moncayo J, et al: Neurocysticercosis. Neurology 41:462-463, 1991

2. Allcut DA, Coulthard A: Neurocysticercosis: regression of a fourth ventricular cyst with praziquantel. J Neurol Neurosurg Psychiatry 54:461-462, 1991

3. Amar AP, Ghosh S, Apuzzo MLJ: Treatment of central nervous system infections: a neurosurgical perspective. Neuroimaging Clin N Am 10:445-459, 2000

4. Apuzzo MLJ, Dobkin WR, Zee CS, et al: Surgical considerations in treatment of intraventricular cysticercosis. An analysis of 45 cases. J Neurosurg 60:400-407, 1984

5. Bandres JC, White AC Jr, Samo T, et al: Extraparenchymal neurocysticercosis: report of five cases and review of management. Clin Infect Dis 15:799-811, 1992

6. Beaver PC, Jung RC, Cupp EW: Clinical Parasitology, ed 9. Philadelphia: Lea \& Febiger, 1984, pp 505-543

7. Bello Martinez E, de Górgolas Hernández-Mora M, Albisua Sanchez J, et al: Neurocisticercosis en un hospital terciario. Nuevos avances en el diagnóstico y tratamiento. Rev Clin Esp 197:604-610, 1997

8. Beskonakli E, Cayli S, Turgut M, et al: Primary giant granulomatous basal meningitis: an unusual presentation of tuberculosis. Childs Nerv Syst 14:79-81, 1998

9. Bonametti AM, Baldy JL, Bortoliero AL, et al: Neurocisticercose com quadro clínico inicial de meningite aguda. Rev Inst Med Trop Sao Paulo 36:27-32, 1994

10. Botero D: Taeniasis, in Goldsmith R, Heyneman D (eds): Tropical Medicine and Parasitology. Norwalk, CT: Appleton \& Lange, 1989, pp 490-500

11. Carpio A, Placencia M, Santillán F, et al: A proposal for classification of neurocysticercosis. Can J Neurol Sci 21:43-47, 1994

12. Carpio A, Santillan F, Leon P, et al: Is the course of neurocysticercosis modified by treatment with anhelminthic agents? Arch Intern Med 155:1982-1988, 1995

13. Chang $\mathrm{KH}$, Lee JH, Han $\mathrm{MH}$, et al: The role of contrastenhanced MR imaging in the diagnosis of neurocysticercosis. AJNR 12:509-512, 1991

14. Colli BO, Martelli N, Assirati JA Jr, et al: Results of surgical treatment of neurocysticercosis in 69 cases. J Neurosurg 65: 309-315, 1986 
15. Colli BO, Pereira CU, Assirati Jr JA, et al: Isolated fourth ventricle in neurocysticercosis: pathophysiology, diagnosis, and treatment. Surg Neurol 39:305-310, 1993

16. Couldwell WT, Chandrasoma P, Apuzzo MLJ, et al: Third ventricular cysticercal cyst mimicking a colloid cyst: case report. Neurosurgery 37:1200-1203, 1995

17. Couldwell WT, Zee CS, Apuzzo ML: Definition of the role of contemporary surgical management in cisternal and parenchymatous cysticercosis cerebri. Neurosurgery 28:231-237, 1991

18. Cuetter AC, Andrews RJ: Intraventricular neurocysticercosis, in Singh G, Prabhakar S (eds): Taenia Solium Cysticercosis. Oxford, UK: CABI Publishing, 2002, pp 199-210

19. Cuetter AC, Garcia-Bobadilla J, Guerra LG, et al: Neurocysticercosis: Focus on intraventricular disease. Clin Infect Dis 24: 157-164, 1997

20. de Morais-Rego SF, Latuf NL: Cisticercose do quarto ventrículo simulando neoplasia da fossa posterior à cintillografia cerebral. Relato de um caso. Arq Neuropsiquiatr 36:371-374, 1978

21. Del Brutto OH, Rajshekhar V, White AC Jr, et al: Proposed diagnostic criteria for neurocysticercosis. Neurology 57: 177-183, 2001

22. Del Brutto $\mathrm{OH}$, Sotelo J: Albendazole therapy for subarachnoid and ventricular cysticercosis. J Neurosurg 72:816-817, 1990

23. Del Brutto OH, Sotelo J, Roman GC: Therapy for neurocysticercosis: a reappraisal. Clin Infect Dis 17:730-735, 1993

24. Del Brutto OH, Wadia NH, Dumas M, et al: Proposal of diagnostic criteria for human cysticercosis and neurocysticercosis. J Neurol Sci 142:1-6, 1996

25. Duplessis E, Dorwling-Carter D, Vidaillet M, et al: Neurocysticercose intraventriculaire. A propos de trois observations. Neurochirurgie 34:275-279, 1988

26. Estañol B, Kleriga E, Loyo M, et al: Mechanisms of hydrocephalus in cerebral cysticercosis: implications for therapy. Neurosurgery 13:119-123, 1983

27. Garcia HH, Gilman RH, Horton J, et al: Albendazole therapy for neurocysticercosis: a prospective double-blind trial comparing 7 versus 14 days of treatment. Cysticercosis Working Group in Peru. Neurology 48:1421-1427, 1997

28. Hanlon KA, Vern BA, Tan WS, et al: MRI in intraventricular neurocysticercosis: a case report. Infection 16:242-244, 1988

29. Joubert J: Cysticercal meningitis - a pernicious form of neurocysticercosis which responds poorly to praziquantel. S Afr Med J 77:528-530, 1990

30. Kanamalla US, Ibarra RA, Jinkins JR: Imaging of cranial meningitis and ventriculitis. Neuroimaging Clin N Am 10: 309-331, 2000

31. Lobato RD, Lamas E, Portillo JM, et al: Hydrocephalus in cerebral cysticercosis. Pathogenic and therapeutic considerations. J Neurosurg 55:786-793, 1981

32. Madrazo I, Garcia-Renteria JA, Sandoval M, et al: Intraventricular cysticercosis. Neurosurgery 12:148-152, 1983

33. McCormick GF: Cysticercosis-review of 230 patients. Bull Clin Neurosci 50:76-101, 1985

34. Monteiro L, Almeida-Pinto J, Stocker A, et al: Active neurocysticercosis, parenchymal and extraparenchymal: a study of 38 patients. J Neurol 241:15-21, 1993

35. Proano JV, Madrazo I, Avelar F, et al: Medical treatment for neurocysticercosis characterized by giant subarachnoid cysts. $\mathbf{N}$ Engl J Med 345:879-885, 2001

36. Proano JV, Madrazo I, Garcia L, et al: Albendazole and praziquantel treatment in neurocysticercosis of the fourth ventricle. J Neurosurg 87:29-33, 1997
37. Puri V, Sharma DK, Kumar S, et al: Neurocysticercosis in children. Indian Pediatr 28:1309-1317, 1991

38. Rogel-Ortiz F, Vera-Pedro M: Meningitis cisticercosa. Gac Med Mex 133:301-305, 1997

39. Salazar A, Sotelo J, Martinez H, et al: Differential diagnosis between ventriculitis and fourth ventricle cyst in neurocysticercosis. J Neurosurg 59:660-663, 1983

40. Salinas R, Counsell C, Prasad K, et al: Treating neurocysticercosis medically: a systematic review of randomized, controlled trials. Trop Med Int Health 4:713-718, 1999

41. Sandoval M, Madrazo I, Garcia-Renteria JA, et al: Obstruction of the ventricular catheter of a CSF shunt system due to the own cyst of Taenia solium. Arch Invest Med 21:95-98, 1990

42. Schantz PM, Sarti E, Plancarte A, et al: Community-based epidemiological investigations of cysticercosis due to Taenia solium: comparison of serological screening tests and clinical findings in two populations in Mexico. Clin Infect Dis 18: 879-885, 1994

43. Shah GV: Central nervous system tuberculosis: imaging manifestations. Neuroimaging Clin N Am 10:355-374, 2000

44. Shandera WX, White AC Jr, Chen JC, et al: Neurocysticercosis in Houston, Texas. A report of 112 cases. Medicine 73:37-52, 1994

45. Sotelo J: Treatment of brain cysticercosis. Surg Neurol 48: 110-112, 1997

46. Sotelo J, Penagos P, Escobedo F, et al: Short course of albendazole therapy for neurocysticercosis. Arch Neurol 45: 1130-1133, 1988

47. Stern WE: Neurosurgical considerations of cysticercosis of the central nervous system. J Neurosurg 55:382-389, 1981

48. Suastegui Roman RA, Soto-Hernandez JL, Sotelo J: Effects of prednisone on ventriculoperitoneal shunt function in hydrocephalus secondary to cysticercosis: a preliminary study. J Neurosurg 84:629-633, 1996

49. Vasconcelos D, Cruz-Segura H, Mateos-Gomez H, et al: Selective indications for the use of praziquantel in the treatment of brain cysticercosis. J Neurol Neurosurg Psychiatry 50: 383-388, 1987

50. Wilson M, Bryan RT, Fried JA, et al: Clinical evaluation of the cysticercosis enzyme-linked immunoelectrotransfer blot in patients with neurocysticercosis. J Infect Dis 164:1007-1009, 1991

51. Wray SD, Ellis TL, Bianco S: Migratory neurocysticercosis mimicking a third ventricular colloid cyst. Case report. J Neurosurg 95:122-123, 2001

52. Zee CS, Go JL, Kim PE, et al: Imaging of neurocysticercosis. Neuroimaging Clin N Am 10:391-407, 2000

53. Zee CS, Segall HD, Apuzzo MLJ, et al: Intraventricular cysticercal cysts: further neuroradiologic observations and neurosurgical implications. AJNR 5:727-730, 1984

54. Zee CS, Segall HD, Destian S, et al: MRI of intraventricular cysticersosis: surgical implications. J Comput Assist Tomogr 17:932-939, 1993

Manuscript received January 15, 2002.

Accepted in final form May 24, 2002.

Address reprint requests to: Albert C. Cuetter, M.D., Division of Neurology, Texas Tech University Health Sciences Center, El Paso, Texas. email: neueacc@ttuhsc.edu. 